\title{
Comportamento da produção de espécies reativas de oxigênio em miocárdio de ratos submetidos a treinamento de baixa intensidade em diferentes temperaturas
}

\author{
Jayme Netto Jr. ${ }^{1}$, Domingo Marcolino Braile², Rúbens Cecchini ${ }^{3}$ Antonio Carlos Cicogna ${ }^{4}$, Flávia Alessandra Guarnier ${ }^{3}$, \\ Carlos Marcelo Pastre1, Silvio Assis de Oliveira Jr. ${ }^{4}$, Mário Sugisaki e Eliane Cristina Pastre ${ }^{5}$
}

\section{RESUMO}

Introdução: A prática de exercício físico proporciona aumento da produção de espécies reativas de oxigênio (ERO) resultantes do metabolismo aeróbio e, gera uma quantidade significativa de calor, em conseqüência da produção de energia, resultando em sobrecarga orgânica. A associação entre ERO e exercício, e entre exercício e variações da temperatura ambiente têm sido estudadas, contudo, há escassez de informações que considere a associação entre produção de radicais livres no miocárdio e atividade física em temperatura elevada. Objetivo: Comparar a produção de ERO em miocárdio de ratos submetidos ao treinamento de baixa intensidade em diferentes temperaturas. Métodos: Foram utilizados 20 ratos Wistar, machos, jovens, peso (250 a 280g), divididos em quatro grupos: $G_{1}(n=5)$ expostos ao treinamento e calor $\left(39^{\circ} \pm 1 \mathrm{C}\right) ; \mathrm{G}_{2}(\mathrm{n}=5)$ expostos somente ao calor durante $\mathrm{O}$ mesmo período de $G_{1}$, sem treinamento; $G_{3}(n=5)$ expostos ao treinamento em temperatura ambiente $\left(22^{\circ} \pm 1 \mathrm{C}\right) ; \mathrm{G}_{4}(n=5)$ expostos à temperatura ambiente sem treinamento. $\mathrm{O}$ treinamento foi realizado em esteira rolante climatizada por cinco semanas, evoluindo 5 minutos a cada duas sessões finalizando em 60 minutos em baixa intensidade $8 \mathrm{~m} / \mathrm{min}$. O ambiente foi controlado entre $39 \pm 1^{\circ} \mathrm{C}$ e $22 \pm 1^{\circ} \mathrm{C}$ e entre 40 e $60 \%$ de umidade relativa. $A$ lipoperoxidação foi avaliada por Quimiluminescência (QL). A análise dos dados foi realizada a partir do teste Two Way ANOVA para análise da QL e t de student para a Capacidade Antioxidante Total (TRAP). Resultados: A análise da QL revelou uma curva de emissão de luz significantemente mais baixa para o grupo exposto ao exercício em normotermia comparado aos sedentários mantidos no calor. A análise da TRAP mostrou diminuição em todos os grupos experimentais em relação ao $\mathrm{G}_{4}$. Conclusão: Concluiu-se que houve níveis menores de produção de ERO nos grupos submetidos somente ao calor ou somente ao exercício.

1. Departamento de Fisioterapia, FCT/UNESP, Presidente Prudente, SP.

2. Departamento de Cardiologia e Cirurgia Cardiovascular, FAMERP, S.J. Rio Preto, SP.

3. Departamento de Ciências Patológicas, UEL, Londrina, PR.

4. Departamento de Clínica Médica, FM/UNESP, Botucatu, SP

5. Programa de Pós-Graduação em Ciências da Saúde, FAMERP, S.J. Rio Preto, SP.

Aceito em 13/6/07

Endereço para correspondência: Jayme Netto Jr., Rua José Vieira, 145, Resiedencial Damha I - 19053-360 - Presidente Prudente, SP.

E-mail: jn@fct.unesp.br

\author{
Palavras-chave: Estresse oxidativo. Exercício físico. Calor \\ Keywords: \\ Oxidative stress. Physical exercise. Heat.
}

\section{ABSTRACT \\ Behavior of oxygen reactive species production in myocardium of rats submitted to low intensity training under different temperatures}

Introduction: The practice of physical exercise causes increase in production of oxygen reactive species (ORS), derived from the aerobic metabolism, creating a significant amount of heat due to the energy production resulting in organic overload. The associations between ORS and exercise, as well as exercise and air temperature variations have been studied; however there is a lack of information on the scientific literature concerning the association between the myocardium free radicals production and physical activity under high temperature. Objective: The goal of this study was to compare the myocardium ORS production in rats submitted to low intensity training at different temperatures. Methodology: Twenty Wistar young male rats, with weight rage from 250 and 280 grams were used. They were divided in four groups: $(G, n$ = 5) exposed to training and heat $\left(39 \pm 1^{\circ} \mathrm{C}\right) ;\left(G_{2} n=5\right)$ exposed to heat without training; $\left(G_{3} n=5\right)$ exposed to training and air temperature $\left(22 \pm 1^{\circ} \mathrm{C}\right) ;\left(G_{4} n=5\right)$ exposed to air temperature without training. The training was performed on a treadmill in a controlled temperature room during 5 weeks, increasing 5 minutes every two sessions, reaching a total of 60 minutes under low intensity effort, $8 \mathrm{~m} / \mathrm{min}$. Room temperature was controlled between $39 \pm$ $1^{\circ}$ and $22 \pm 1^{\circ}$, as well as between 40 to $60 \%$ of relative humidity. Lipoperoxidase was evaluated by Chemiluminescense (OL). Data analysis was accomplished using Two-Way ANOVA test. Results: The $Q L$ analysis results revealed a light emission curve significantly lower for the animals exposed to room temperature exercise, compared to the sedentary ones kept under heat. TRAP analysis has showed a decrease in every experimental group in relation to $G_{4}$. Conclusion: It can be concluded that there were lower levels of ORS production in the groups submitted either to heat only or exercise only.

\section{INTRODUÇÃO}

Notadamente, a prática regular de exercícios físicos tem sido amplamente associada à melhora nos padrões de saúde de seus praticantes. Desta forma, acredita-se que sua prática regular, represente papel fundamental na prevenção de doenças crônicodegenerativas ao minimizarem os efeitos de boa parte dos fatores de risco das mesmas ${ }^{(1)}$.

Entretanto, quando praticado em ambientes inadequados, sem orientação e controle profissional, pode proporcionar danos à saúde de seus praticantes como: envelhecimento precoce, lesões nos músculos esqueléticos, aumento no risco de problemas car- 
díacos ou até a morte súbita, como observado em atletas e indivíduos fisicamente ativos durante ou imediatamente após a execução de exercícios físicos ${ }^{(2-3)}$. Destaca-se, além disso, a ocorrência de lesões oxidativas em biomoléculas devido à prática de exercícios físicos, conforme observado em estudos experimentais e em humanos ${ }^{(4-5)}$.

Como conseqüência da vida aeróbia, o organismo humano produz continuamente radicais livres conhecidos como espécies reativas do oxigênio $(E R O)^{(4)}$. Define-se como estresse oxidativo a alteração no estado de equilíbrio entre produção de ERO e sua remoção pelos sistemas antioxidantes celulares(6). O aumento no consumo de oxigênio, por meio da ativação das diferentes vias metabólicas, durante ou após exercícios físicos, resulta no aumento de $\mathrm{ERO}^{(7-8)}$

Ademais, durante a prática de exercícios físicos, uma quantidade significativa de calor é gerada pelo organismo como subproduto do metabolismo energético. Com uma mesma carga de trabaIho, esta prática, em ambientes quentes, promove aumento na freqüência cardíaca e no débito cardíaco, assim como aumento na temperatura interna e cutânea, comparativamente com o mesmo exercício realizado em ambientes frios. Neste sentido, exercícios físicos em ambientes quentes promovem aumento na concentração sanguínea de lactato e existem evidências de depleção mais rápida do glicogênio muscular ${ }^{(9)}$. Estudos relatam que altas temperaturas teciduais induzem alterações estruturais e funcionais nas proteínas celulares envolvidas no transporte eletrolítico por meio da membrana celular e do retículo sarcoplasmático e durante a respiração mitocondrial, antecipando a fadiga muscular com redução da performance atlética(10-11).

As características metabólicas aeróbicas do músculo cardíaco são diferentes dos outros tecidos e sua capacidade de gerar energia por processos anaeróbios é limitada. Enquanto que, em repouso, alguns tecidos utilizam apenas a quarta parte do oxigênio disponível na circulação, o miocárdio capta cerca de 70\% de oxigênio do fluxo coronariano, uma vez que no tecido cardíaco há uma maior concentração de mitocôndrias, tornando-o altamente adaptado para o catabolismo lipídico como fonte primária da ressíntese de adenosina trifosfato ${ }^{(12)}$

Dessa forma, durante atividade física, há incremento entre 10 e 20 vezes no consumo total de oxigênio do organismo e aumento variando entre 100 e 200 vezes na captação de oxigênio pelo tecido muscular, favorecendo uma produção maior de ERO, sugerindo, portanto, uma possível associação entre exercícios de endurance e estresse oxidativo no miocárdio(13).

A partir de dados apontados pela literatura, pode-se dizer que existe estreita relação entre exercício físico, estresse oxidativo e temperaturas elevadas, contudo, há escassez de dados na literatura considerando a associação entre produção de ERO em miocárdio de ratos e treinamento físico em diferentes temperaturas ambiente. Em função disso, o objetivo do presente estudo foi analisar a produção das Espécies Reativas de Oxigênio em miocárdio de ratos submetidos ao treinamento físico de baixa intensidade em diferentes temperaturas.

\section{MÉTODOS}

Os estudos realizados foram apreciados e aprovados pela Comissão de Ética e Experimentação Animal (CEAA) (Proc. 921/2005) da Faculdade de Medicina de São José do Rio Preto (FAMERP). Caracterizam-se como experimental prospectivo controlado, apresentando como variável dependente, a produção das ERO, e como independentes, a umidade relativa do ar, temperatura ambiente e intensidade de esforço físico.

Para realização do estudo foram utilizados 20 ratos machos, da linhagem Wistar (Rattus novergicus, var. albina, Rodentia, Mammalia), com 60 dias de idade, pesando entre 250 e 280 gramas. Os animais foram mantidos em gaiolas plásticas coletivas, con- tendo no máximo cinco animais cada gaiola em temperatura média de $22 \pm 2^{\circ} \mathrm{C}$ e ciclo claro/escuro de 12 horas. A alimentação foi baseada em ração padrão e água de torneira ad libitum.

\section{Protocolo experimental}

Os animais foram divididos em quatro grupos, sendo dispostos da seguinte forma: $\mathbf{G}_{\mathbf{1}}(\mathbf{n}=\mathbf{5})$ - animais expostos ao treinamento e calor $\left(39^{\circ} \pm 1 \mathrm{C}\right) ; \mathbf{G}_{\mathbf{2}}(\mathbf{n}=\mathbf{5})$ - animais expostos somente ao calor durante o mesmo período de $\mathbf{G}_{\mathbf{1}}$, sem treinamento; $\mathbf{G}_{\mathbf{3}}(\mathbf{n}=\mathbf{5})$ animais expostos ao treinamento em temperatura ambiente (22。 $\pm 1 \mathrm{C}) ; \mathbf{G}_{\mathbf{4}}(\mathbf{n}=\mathbf{5})$ - animais expostos à temperatura ambiente sem treinamento o mesmo período de tempo dos demais, Todos os animais foram eutanasiados por decapitação no tempo correspondente a 72 horas após a última sessão de treinamento e de exposição ao calor e normotermia. Os corações foram rapidamente removidos, lavados em $\mathrm{KCl}(1,15 \%)$, e estocados em Nitrogênio líquido por até 80 dias.

O treinamento dos animais foi realizado a partir de corrida em esteira climatizada de acordo com modelo Andrew ${ }^{(14)}$, por um período de seis semanas, com cinco sessões semanais (de segunda à sexta-feira).

$\mathrm{Na}$ primeira semana, foi feita a adaptação progressiva para o ambiente da esteira climatizada, iniciando com 15 minutos de treinamento, com aumento de cinco minutos a cada duas sessões até completar 25 minutos no quinto dia da semana. Tal fase de adaptação foi utilizada, também, para exclusão dos animais que se recusavam a correr.

A partir da segunda semana, com os grupos definidos, utilizouse o mesmo princípio de cargas crescentes, iniciou-se o treinamento propriamente dito, com 25 minutos de corrida e, a cada duas sessões, era acrescido cinco minutos no tempo de realização de treinamento, até completar 85 minutos ao final da sexta semana. A velocidade da esteira foi mantida em $8 \mathrm{~m} / \mathrm{min}$, caracterizando o exercício de baixa intensidade, segundo sugerem CarvaIho et al.(15). Para a simulação do ambiente climatizado foi acoplado um gabinete, envolvendo as cinco pistas. A elevação e redução da temperatura foram feitas, respectivamente, por meio de um resistor de aquecimento e refrigerador de ar, ligados ao sistema de ventilação do gabinete. A umidade relativa do ar foi mantida entre 40 e $60 \%$ por meio de spray manual. Para o controle destas variáveis foram colocados sensores na parte interna do gabinete, ligados a um termômetro digital (ICEL HT-208, Manaus - Brasil), fixado na parte externa.

\section{Obtenção de homogenato e sobrenadante do miocárdio de rato}

No momento das análises, o coração de cada animal foi pesado e transferido para uma solução de tampão Fosfato de Potássio Monobásico $\left(\mathrm{K}_{2} \mathrm{HPO}_{4}\right) 10 \mathrm{mM}$ em Cloreto de Sódio $(\mathrm{NaCl})$ a 0,9\%, $\mathrm{pH} 7,4$ na proporção de $10 \mathrm{ml}$ de solução para cada $100 \mathrm{mg}$ de tecido. Cada amostra foi então submetida a homogeneizador tipo ultra-turrax (Fabricante: Marconi, Piracicaba - SP, Brasil), sob banho de gelo. Os tecidos foram homogeneizados de igual maneira, em 5 tempos de 30 segundos, com 1 minuto de intervalo. Utilizou-se homogenato total para medida da quimiluminescência, e o sobrenadante proveniente de uma cemtrifugação a 11.000rpm, por 15 minutos a $4^{\circ} \mathrm{C}$, para a Capacidade Antioxidante Total (TRAP).

\section{Quimiluminescência estimulada por hidroperóxido de tert-butil}

Para avaliar os níveis de peróxidos lipídicos no miocárdio dos animais submetidos ao protocolo foi utilizado o método de Quimiluminescência (QL) induzida por tert-butil hidroperóxido descrito por Goonzales-Flecha et al. ${ }^{(16)}$. As amostras - $(875 \mu \mathrm{L})$ do homogenato total - após incubação com tampão $\mathrm{KH}_{2} \mathrm{PO}_{4}$, a $30^{\circ} \mathrm{C}$ por 5 minutos, ao abrigo da luz, foram submetidas à reação com tertbutil hidroperóxido (concentração final de $6 \mathrm{mM}$ ), em luminômetro 
(Turner Designs TD-20/20 Califórnia, EUA), com captação de resposta entre 300-650nm, durante 40 minutos. $O$ aumento da emissão de fótons indica a presença de lipoperóxidos pré-formados na membrana do miocárdio, revelando o ataque de ERO às mesmas. As curvas foram obtidas por meio da interpolação de 40 pontos (minuto a minuto) a partir da curva original. Os resultados foram expressos em Unidades Relativas de Luz (URL)/g de tecido.

\section{Determinação da capacidade antioxidante total (TRAP) por luminescência}

A TRAP foi avaliada por quimiluminescência segundo a técnica descrita por Repetto et al.(177). Essa técnica avalia os níveis de antioxidantes totais hidrossolúveis de um tecido, principalmente os de baixo peso molecular, por meio de uma reação contendo $20 \mu \mathrm{M}$ de 2,2-azo-bis(2-amidinopropano) e 200 $\mu \mathrm{M}$ de luminol. A adição de $70 \mu \mathrm{L}$ do sobrenadante diminui a luminescência produzida a níveis basais por um período (tempo de indução - Tind) proporcional à quantidade de antioxidante presente na amostra, até que atinja o nível de geração de luz padrão. O sistema foi calibrado com trolox e a comparação entre o $t$ ind da reação com o trolox e o $t$ ind da reação com amostra permitiram obter valores de TRAP em $\mu \mathrm{M}$ de trolox.

O Pico de emissão da TRAP foi calculado, de acordo com Nugyen-Khoa et al.(18), expresso em URL, possibilitando a análise total das curvas.

\section{Análise estatística}

As análises descritivas de todas as variáveis estudadas estão apresentadas como médias e erros-padrão da média (SEM). Para a comparação das variáveis, Velocidade Inicial $\left(V_{0}\right)$, Pico de Emissão, T.ind, Rate e TRAP, segundo os fatores, treinamento e temperatura, considerou-se a técnica de Analise de Variância para um modelo experimental para dois fatores correspondentes (esquema fatorial $2 \times 2$ ) no delineamento inteiramente casualizado. Como Post Hoc utilizou-se o teste de Tukey para comparações múltiplas entre pares de médias. Todas as comparações foram analisadas em nível de $5 \%$ de significância. Para análise da QL, foi utilizado o mesmo procedimento estatístico, sendo sua representação gráfica expressa a partir do programa Graph Pad Prism 4. A análise de regressão linear foi utilizada para obter a velocidade inicial $\left(V_{0}\right)$ da curva de $\mathrm{QL}$, unindo-se os pontos inicial e máximo de leitura da curva média QL-tempo.

\section{RESULTADOS}

Observa-se na tabela 1 que o pico de emissão de luz, no grupo de animais expostos ao treinamento em temperatura ambiente, foi significativamente menor em relação aos demais grupos. Notou-se também, que no grupo de animais sedentários expostos ao calor, os valores de pico foram menores comparados aos sedentários em temperatura ambiente $(P<0,007)$. Para a variável velocidade inicial da reação de $\mathrm{QL}\left(V_{0}\right)$ a média dos valores encontrados nos animais do grupo treinado em temperatura ambiente revelou uma curva de tempo significativamente menor em relação aos grupos de animais não treinados expostos ao calor e à temperatura ambiente.

TABELA 1

Valores da média e do erro padrão da Quimiluminescência (QL) estimulada por hidroperóxido de tert-Butil $(t-\mathrm{BOOH})$, em músculo cardíaco de ratos submetidos à eutanásia $72 \mathrm{~h}$

\begin{tabular}{ccc}
\hline Grupos & Vo (URL/min.) & Pico de Emissão (URL) \\
$\mathrm{G}_{1}$ & $8,1 \pm 2,1^{\mathrm{b}}$ & $85,21 \pm 5,43^{\mathrm{bc}}$ \\
$\mathrm{G}_{2}$ & $11,7 \pm 1,5^{\mathrm{b}}$ & $40,30 \pm 9,30^{\mathrm{b}}$ \\
$\mathrm{G}_{3}$ & $5,7 \pm 0,12^{\mathrm{a}}$ & $54,36 \pm 3,63^{\mathrm{a}}$ \\
$\mathrm{G}_{4}$ & $9,0 \pm 0,9^{\mathrm{b}}$ & $80,39 \pm 0,79^{\mathrm{c}}$ \\
\hline
\end{tabular}

Nota: A comparação dos resultados entre os grupos para cada variável e a descrição das diferenças estatisticamente significantes dadas por Two Way Anova $(P \leq 0,05)$ são expressas por letras. Entendendo que letras iguais não apontam diferenças sendo que $\mathrm{a}<\mathrm{b}$.
A figura 1 mostra a curva de luminescência, expressa em unidade relativa de luz por grama de tecido, em razão do tempo. Evidencia-se que os animais expostos ao calor sem treinamento apresentam uma curva expressivamente elevada em relação aos expostos ao treinamento em temperatura ambiente $(P<0,001)$.

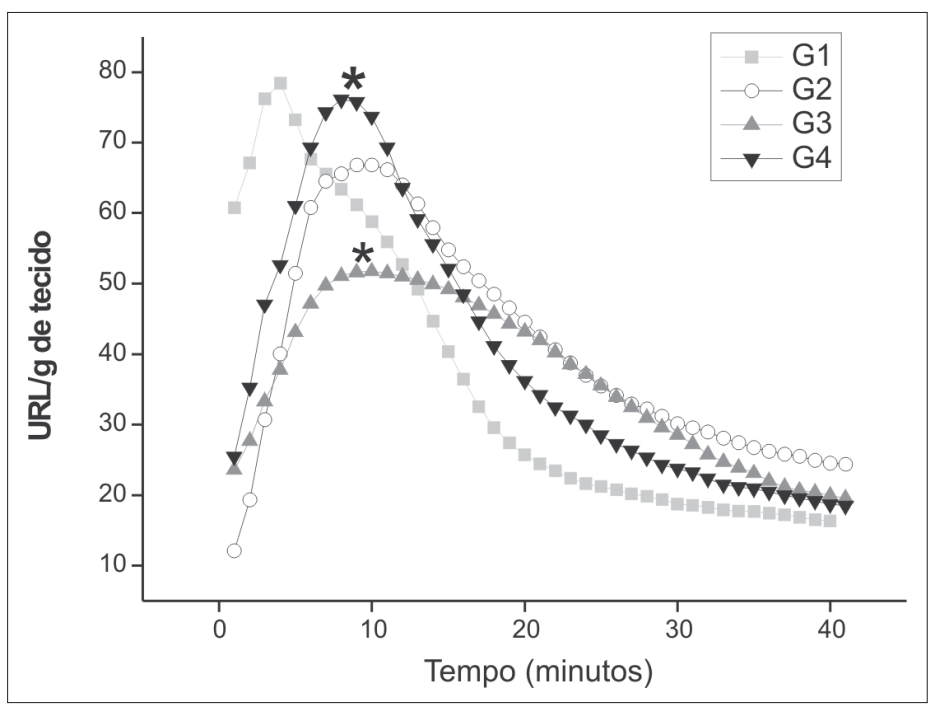

Figura 1 - Curvas obtidas nas análises de Quimiluminescência estimulada por hidroperóxido de tert-Butil (t-BOOH) em músculo cardíaco de ratos submetidos à eutanásia tardia, expressa em unidades relativas de luz por grama de tecido (URL/g tecido), em razão do tempo em minutos, dos A comparação dos resultados entre os grupos e a descrição das diferenças estatisticamente significantes $(P \leq 0,05)$, são expressas por letras descritas na legenda. Letras diferentes representam diferença estatística. As curvas representam 40 pontos extraídos da curva original, e comparados por Two Way Anova.

Os valores das variáveis relativas aos testes da TRAP dos grupos e suas respectivas comparações são mostrados na tabela 2 . Notou-se que para o tempo de indução, o grupo de animais sedentários em temperatura ambiente, apresentou valores estatisticamente maiores em relação aos demais grupos. Tal grupo, na análise do Rate, apresentou valores menores comparados aos expostos ao exercício e calor $(P<0,02)$. Em relação aos valores de Pico de emissão, os sedentários expostos à temperatura ambiente apresentaram valores menores em relação aos expostos ao exercício e calor $(\mathrm{P}<0,02)$ e aos sedentários expostos ao calor $(\mathrm{P}$ $<0,04)$. Notou-se também, que os sedentários expostos ao calor têm valores de pico maiores em relação aos expostos ao exercício e calor $(P<0,04)$. Para análise da TRAP, o grupo de animais sedentários expostos à temperatura ambiente mostrou valores maiores em relação aos demais grupos, revelando diminuição da capacidade antioxidante tanto dos animais expostos somente ao calor, quanto dos animais expostos somente ao exercício, ou de ambos.

\section{TABELA 2}

Valores da média e erro padrão na análise da Capacidade Antioxidante Total (TRAP), dos animais submetidos à eutanásia imediata $72 \mathrm{~h}$

\begin{tabular}{ccccc}
\hline Grupos & $\begin{array}{c}\text { T. ind } \\
\text { (min.) }\end{array}$ & $\begin{array}{c}\text { Rate } \\
\text { (URL/min.) }\end{array}$ & $\begin{array}{c}\text { Pico } \\
\text { (URL) }\end{array}$ & $\begin{array}{c}\text { TRAP } \\
\text { ( } \boldsymbol{\mu M} \text { Mrolox) }\end{array}$ \\
$\mathrm{G}_{1}$ & $4,554 \pm 0,72^{\mathrm{a}}$ & $113,76 \pm 27,85^{\mathrm{b}}$ & $534,86 \pm 60,70^{\mathrm{a}}$ & $0,589 \pm 00,9^{\mathrm{a}}$ \\
$\mathrm{G}_{2}$ & $5,488 \pm 1,17^{\mathrm{a}}$ & $193,08 \pm 79,87^{\mathrm{ab}}$ & $1026,08 \pm 79,87^{\mathrm{c}}$ & $0,718 \pm 0,15^{\mathrm{a}}$ \\
$\mathrm{G}_{3}$ & $5,943 \pm 0,43^{\mathrm{a}}$ & $109,92 \pm 61,01^{\mathrm{ab}}$ & $527,31 \pm 167,00^{\mathrm{abc}}$ & $0,778 \pm 0,05^{\mathrm{a}}$ \\
$\mathrm{G}_{4}$ & $11,399 \pm 1,96^{\mathrm{b}}$ & $34,86 \pm 4,33^{\mathrm{a}}$ & $272,14 \pm 13,22^{\mathrm{b}}$ & $1,491 \pm 0,26^{\mathrm{b}}$ \\
\hline
\end{tabular}

Nota: Comparação utilizando o teste de Analise de Variância para um modelo experimental para dois fatores correspondentes complementado pelo teste de Tukey para comparações múltiplas entre pares de médias. Todas as comparações foram analisadas em nível de $5 \%$ de significância. Entendendo que letras iguais não apontam diferenças sendo que $\mathrm{a}<\mathrm{b}<\mathrm{c}<\mathrm{d}$. 
A figura 2 mostra os valores da Capacidade Antioxidante Total (TRAP) no miocárdio dos grupos avaliados. Os valores do controle chegaram a 1,49 $\pm 0,3 \mu \mathrm{mol}$ de trolox; uma diminuição significante na capacidade antioxidante foi observada a partir das exposições somente ao calor, somente ao treinamento ou ambos respectivamente, $(0,78 \pm 0,05 ; 0,72 \pm 0,15$; e 0,59 $\pm 0,09 \mu \mathrm{mol}$ de trolox), ( $P$ $<0,05)$. O grupo submetido ao calor e treinamento concomitantemente apontou diminuição mais proeminente, chegando a $51,7 \%$ de redução.

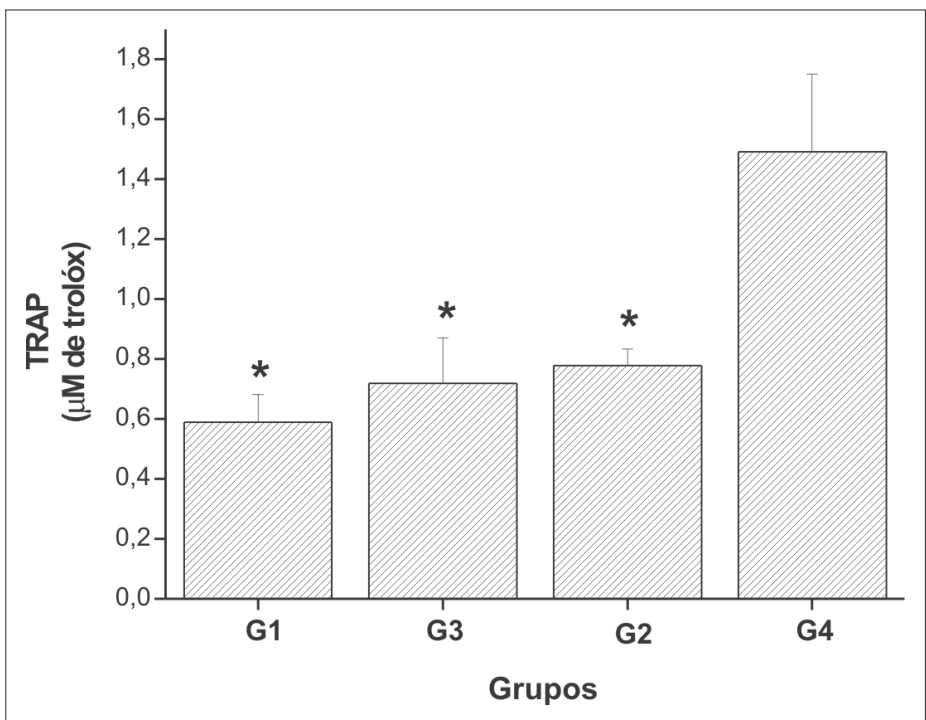

Figura 2 - Gráfico representativo dos valores da Capacidade Antioxidante Total (TRAP) medida por Quimiluminescência em músculo cardíaco de ratos submetidos à eutanásia tardia, expressa em micromoles $(\mu M)$ de trolóx, dos grupos. Comparação utilizando o teste de Analise de Variância para um modelo experimental para dois fatores correspondentes complementado pelo teste de Tukey para comparações múltiplas entre pares de médias. Todas as comparações foram analisadas em nível de $5 \%$ de significância. Todas as comparações foram analisadas em nível de 5\% de significância. ${ }^{*} P \leq 0,05$ em relação ao grupo $G_{4}$.

\section{DISCUSSÃo}

Em relação ao contexto empírico, experiências práticas vividas a partir das observações de sujeitos submetidos ao exercício físico em condição de calor intenso, estimularam o interesse pelo assunto. Contudo, constituiu-se, definitivamente em situação-problema, quando da busca por evidências científicas acerca do tema.

Inicialmente, observou-se em estudo recente desenvolvido por Jenkins ${ }^{(19)}$ que discutia a metodologia aplicada para verificação da relação entre exercício físico e estresse oxidativo, poucas citações sobre a utilização dos métodos de QL e TRAP, métodos sensíveis aplicados para estudos desta natureza e que foram os eleitos para realização da presente pesquisa. Além disso, a partir de uma exploração mais aprofundada sobre a relação entre atividade física, exposição ao calor e estresse oxidativo no miocárdio constatou-se ausência de referências.

Dessa forma, entendeu-se como pertinente, elaborar um modelo experimental que se aproximasse da realidade dos praticantes de exercícios físicos em diferentes temperaturas, e empreender investigação sobre o tema. A utilização de animais deu-se pela ação invasiva das análises do coração, não só, pela necessidade de inserir elementos adicionais à escassa literatura científica, mas também, pela possibilidade de contribuir com as ciências da saúde e do esporte, para melhor entendimento de problemas importantes, como danos cardíacos durante a realização de exercícios em ambientes com temperatura elevada, evento comum em países de clima tropical como o Brasil.
A opção pela análise concomitante da QL e do TRAP se faz necessária para avaliar a capacidade antioxidante disponível em cada tecido, atuando como inibidor das lesões detectadas na QL. É o inbalance entre capacidade oxidante/antioxidante que provoca o aumento dos níveis de emissão. Estudos prévios demonstraram que a utilização de $\mathrm{QL}$, sendo um método mais sensível, apresenta maior correlação com o método da reação do MDA com TBARS, o mais amplamente empregado para detecção de lipoperoxidos de membrana(16-17,20).

Apesar dos cuidados com o desenvolvimento do estudo, alguns pontos metodológicos mostraram-se frágeis e merecem discussão. Em relação ao protocolo experimental, constatou-se que o número de animais foi pequeno para uma técnica extremamente sensível, o que pode provocar aumento dos valores de erro padrão. Para futuros estudos, sugere-se um número maior de animais por grupo.

Neste estudo não se incluiu um parâmetro objetivo de determinação de intensidade de exercício, como o lactato ou consumo máximo de oxigênio, apesar de todo o protocolo ter sido embasado na literatura, como sugeridos por Carvalho et al.(15) e Wisløff et al.(21) que observaram a correlação entre os valores das variáveis citadas e a intensidade de esforço a partir da velocidade aplicada ao protocolo.

Outro aspecto limitante foi que as lesões não foram avaliadas imediatamente após a última sessão de treinamento, nem após um período maior que três dias, para que se observasse a evolução do processo de recuperação. Destaca-se, também, a ausência de um grupo para observação do comportamento das ERO após exposição ao exercício agudo em diferentes temperaturas.

Em relação aos achados, notou-se que o pico de emissão dos animais expostos ao calor e exercício foi maior em relação aos demais, sugerindo maior estresse sofrido pelo tecido dos animais desse grupo. Tais resultados concordam com os estudos de McAnulty et al.(22) realizados com seres humanos a partir de exercícios de baixa intensidade realizados em esteira rolante e ambiente quente e de Mills et al.(23), analisando cavalos em ambientes de alta temperatura e umidade de ar, cujos parâmetros bioquímicos como concentração plasmática de glutationa oxidada e hidroperóxidos lipídicos apresentaram-se aumentados.

A curva de luminenscência de animais sedentários expostos ao calor mostra menor lipoperoxidação em relação aos grupos expostos ao calor e exercício e, sedentários mantidos em temperatura ambiente, sugerindo efeito condicionante. Como hipótese explicativa para tal evento, apresenta-se a teoria fisiológica da termorregulação conforme Caterina(24). Em síntese, os termorreceptores detectam alterações ambientes e enviam mensagem ao hipotálamo com imediata condição de defesa a partir de alterações do metabolismo basal visando à manutenção da homeostase.

Husain et al.(25) e Taylor et al.(26) corroboram os achados desta pesquisa em relação ao efeito condicionante do exercício crônico contra os danos causados pelo estresse oxidativo. Na presente pesquisa observou-se, no grupo exposto ao exercício em temperatura ambiente, os menores indicadores quando comparado aos demais grupos.

A diferença significante anotada a partir da análise das curvas de luminescência entre os animais sedentários expostos ao calor e os treinados em temperatura ambiente sugere que a exposição crônica ao calor, apesar de condicionante, conforme o discutido, pode ser um fator gerador de estresse mesmo na ausência de exercício.

Em relação à análise da TRAP, observou-se diferença significante entre controle e demais grupos. Tais resultados não apresentaram coerência com o estresse notado nas curvas de quimiluminescência. A recuperação dos animais sedentários expostos ao calor e treinados em temperatura ambiente deveriam apresentar melhores níveis de recuperação dos antioxidantes não solúveis. 
Todavia, sabe-se que, segundo Halliwell e Gutteridge(27), a análise de antioxidantes totais avalia os antioxidantes de baixo peso molecular, como $\alpha$-tocopherol, ácido úrico, ácido ascórbico, glutathiona total, dentre outros, que representam aproximadamente 30\% dos antioxidantes avaliados pelo tipo de metodologia empregada. Não se pode, ainda, descartar que a redução dos níveis basais de injúria oxidativa observada nos animais experimentais seja resultado de um consumo de antioxidantes, como se nota nesses animais, em relação ao controle.

Os resultados sugerem repercussões sobre implicações práticas acerca do tema. Não houve recuperação das lesões provocadas pela exposição ao calor associada ao exercício em período de três dias, indicando a necessidade de cuidado para realização de atividades físicas, mesmo que de baixa intensidade de esforço em ambientes quentes. No entanto, a exposição sistematizada ao calor, nos animais sedentários, gerou um efeito cardioprotetor, sugerindo que o calor, em si, pode ser um aliado no efeito condicionante em sujeitos com limitações funcionais à prática de exercícios físicos. Todavia, estudos clínicos em seres humanos são

\section{REFERÊNCIAS}

1. Wielenga RP, Coats ASJ, Mosterd WL, Huisveld IA. The role of exercise training in chronic heart failure. Heart. 1997;78:431-36.

2. Barriales VR, Moris T, Penas LM. Sudden death, sports activities and coronary artery anomalies. Rev Esp Cardiol. 2002;55(10):1105-6.

3. Suarez M, Aguilera B. Causes of sudden death during sports activities in Spain. Rev Esp Cardiol. 2002;55(4):347-58.

4. Schneider DC, De Oliveira RA. Radicais livres de oxigênio e exercício: mecanismos de formação e adaptação ao treinamento físico. Rev Bras Med Esporte. 2004;10(4):308-12.

5. Córdova A, Navas JF. Os radicais livres e o dano muscular produzido pelo exercício: papel dos antioxidantes. Rev Bras Med Esporte. 2000;6(5):204-8.

6. Finkel T. Oxidant signals and oxidative stress. Current Opinion in Cell Biology. 2003;15:247-54.

7. El'Chaninova SA, Smagina IV, Korenyak NA, Varshavskii BY. Lipid peroxidation and antioxidant enzyme activity in response to interval hypoxic training. Human Physiology. 2003;29(3):324-7

8. Souza Jr PT, De Oliveira RP, Pereira B. Exercício físico e estresse oxidativo: Efeitos do exercício físico intenso sobre a Quimiluminescência rinária e malondialdeído plasmático. Rev Bras Med Esporte. 2005;11(1):91-6.

9. Rodrigues LOC, Oliveira A, Lima NRV, Machado-Moreira CA. Heat storage rate and acute fatigue in rats. Braz J Med Biol Res. 2003;36(1):131-5.

10. González-Alonso J, Teller C, Andersen SL, Jensen FB, Hyldig T, Nielsen B. Influence of body temperature on the development of fatigue during prolonged exercise in the heat. J Appl Physiol. 1999;86(3):1032-9.

11. Hunter AM, St Clair Gibson A, Mbambo Z, Lambert MI, Noakes TD. The effects of heat stress on neuromuscular activity during endurance exercise. Pflugers Arch. 2002;444(6):738-43.

12. Atalay M, Sen CK. Physical exercise and antioxidant defenses in the heart. Ann NY Acad Sci. 1999;874:169-77.

13. Mastaloudis A, Leonard SW, Traber MG. Oxidative stress in athletes during extreme endurance exercise. Free Rad Biol Med. 2001;31(7):911-22.

14. Andrew RJ. Treadmill for small laboratory animals. Journal of Applied Physiology. 1965;20(3):572-4

15. Carvalho JF, Masuda OM, Pompeu AMSF. Method of diagnosis and control of aerobic training in rats basead on lactate threshold. Comparative Biochemistry and Physiology. 2005;140(4):409-13. necessários para extrapolar os achados da presente pesquisa e comprovar tal hipótese.

Concluiu-se, a partir dos objetivos propostos, que os níveis de lipoperóxidos pré-formados foram menores nos animais submetidos somente ao calor ou somente ao treinamento e maiores nos animais submetidos ao calor associado ao exercício. No que se refere à proteção antioxidante, os perfis de lesão dos grupos experimentais foram reforçados pela diminuição de antioxidantes solúveis em relação ao controle.

\section{AGRADECIMENTOS}

Agradecemos à Fundação para o Desenvolvimento da Unesp (FUNDUNESP) por fomentar integralmente o experimento possibilitando sua idealização e realização.

Todos os autores declararam não haver qualquer potencial conflito de interesses referente a este artigo.
16. Gonzales-Flecha B, LIsuy S, Boveris A. Hidroperoxide initiated chemiluminescence: an assay for oxidative stress in biopsies of heart, liver and muscle. Free radical Biology and Medicine. 1991;10:93-100.

17. Repeto M, Reides C, Carretero GML, Marta C, Griemberg G, Liesuy S. Oxidative stress in blood of HIVi infected patients. Clin Chimica Acta. 1996;255:107-17.

18. Nguyen-Khoa T, Massy AZ, Witko-Sarsat V, Thévenin M, Touam M, Lambrey G, et al. Critical evaluation of plasma and LDL oxidant-trapping potential in hemodialysis patients. Kidney International. 1999;56:747-53.

19. Jenkins RR. Exercise oxidative stress methodology: critique. Am J Clin Nutr. 2000; 72(2 Suppl):670-4

20. Boveris A, Cadenas E, Reiter R, Filipkowski M, Nakase Y, Chance B. Organ chemiluminescence noninvasive assay for oxidative radical reactions. Proc Natl Acad Sci USA. 1980;77:347-51

21. Wisløff U, Helgerude J, Kemi OJ, Ellingsen $\varnothing$. Intensity-controlled treadmill running in rats: $\dot{V O}_{2 \max }$ and cardiac hypertrophy. Am J Physiol Heart Circ Physiol. $2001 ; 280: 1301-10$.

22. McAnulty SR, McAnulty L, Pascoe DD, Gropper SS, Keith RE, Morrow JD, et al Hyperthermia increases exercice-induced oxidative stress. Int J Sports Med 2005;26(3):188-92

23. Mills PC, Smith NC, Casas I, Harris P, Harris RC, Marlin DJ. Effects of exercise intensity and environmental stress on indices of oxidative stress and iron homeostasis during exercise in the horse. Eur J Appl Physiol. 1996;74:60-6.

24. Caterina MJ. Transient receptor potential ion channels as participants in thermosensation and thermoregulation. Am J Physiol Regul Integr Comp Physiol. 2007;292(1):64-76

25. Husain K, Hazelrigg SR. Oxidative injury due to chronic nitric oxide synthase inhibition in rat: effect of regular exercise on the heart. Biochim Biophys Acta. 2002; 1587(1):75-82

26. Taylor RP, Ciccoli JT, Starnes JW. Effect of exercise training on the ability of the rat to tolerate hydrogen peroxide. Cardiol Res. 2003;58:575-81.

27. Halliwell B, Gutteridge JMC. Oxidative stress: adaptation, damage, repair and death. In: Halliwell B, Gutteridge JMC, editors. Free radicals in biology and medicine. Oxford: Oxford University Press; 1999. p. 246-350. 